Meta

Journal des traducteurs

Translators' Journal

\title{
Les traducteurs : ces médecins légistes du texte
}

\section{Christian Balliu}

Volume 46, numéro 1, mars 2001

Traduction médicale et documentation / Medical translation and documentation

URI : https://id.erudit.org/iderudit/001961ar

DOI : https://doi.org/10.7202/001961ar

Aller au sommaire du numéro

Éditeur(s)

Les Presses de l'Université de Montréal

ISSN

0026-0452 (imprimé)

1492-1421 (numérique)

Découvrir la revue

Citer cet article

Balliu, C. (2001). Les traducteurs : ces médecins légistes du texte. Meta, 46(1), 92-102. https://doi.org/10.7202/001961ar

\section{Résumé de l'article}

La médecine est une science plurimillénaire soumise à l'évolution de l'histoire. Son discours, dont la terminologie, ne peut donc échapper à l'influence de ses utilisateurs successifs. Le traducteur médical devra donc être conscient que le sociolecte médical n'est pas alimenté par une terminologie objective et rigoureuse, mais qu'il est assujetti à une instabilité aussi synchronique que diachronique. 


\title{
Les traducteurs: ces médecins légistes du texte
}

\author{
CHRISTIAN BALLIU \\ ISIT, Paris, France
}

\begin{abstract}
RÉSUMÉ
La médecine est une science plurimillénaire soumise à l'évolution de l'histoire. Son discours, dont la terminologie, ne peut donc échapper à l'influence de ses utilisateurs successifs. Le traducteur médical devra donc être conscient que le sociolecte médical n'est pas alimenté par une terminologie objective et rigoureuse, mais qu'il est assujetti à une instabilité aussi synchronique que diachronique.
\end{abstract}

\section{ABSTRACT}

An age-old science, medicine has had to follow in the steps of History for millenniums. Little wonder, then, if medical language — and, hence, terminology — has always been influenced, over the years, by its successive users. The medical translator is bound to realize that the medical sociolect, far from feeding on an exact and objective terminology, is in fact prey to synchronic as well as diachronic instability.

\section{MOTS-CLÉS/KEYWORDS}

traduction médicale, terminologie, linguistique, objectivité scientifique, modalisation

\begin{abstract}
Car la médecine étant un compendium d'erreurs successives et contradictoires des médecins, en appelant à soi les meilleurs d'entre eux on a grande chance d'imposer une vérité qui sera reconnue fausse quelques années plus tard. De sorte que croire à la médecine serait la suprême folie, si n'y pas croire n'en était pas une plus grande, car de cet amoncellement d'erreurs se sont dégagées à la longue quelques vérités.
\end{abstract}

MARCEL PROUST, À la recherche du temps perdu, Le côté de Guermantes

On remarque depuis les années 1960 et surtout 1970 une réorganisation et une ventilation toujours plus importantes des disciplines médicales traditionnelles: en sousdomaines dans un premier temps et en spécialités véritablement autonomes dans un second temps. La multiplication des tiroirs et le nombre croissant de chercheurs davantage spécialisés alimentent la recherche et les publications qui la sous-tendent.

Comme le signalait le rédacteur du British Medical Journal (Lock: 1989), il y avait déjà à l'époque de son article entre 20000 et 25000 revues biomédicales de par le monde, dont 15000 "sérieuses», et chaque domaine donnerait naissance à de nouvelles spécialités tous les dix ans approximativement.

Si les nouvelles disciplines sont très ciblées scientifiquement, elles restent éparses sur le plan géographique et sont alimentées par des réseaux de chercheurs aussi intangibles que réels. Ces chercheurs éprouvent donc au minimum un triple besoin de communiquer leurs expériences par le canal de revues particulières. On peut, pour reprendre la classification de Régent (1992: 67), résumer cette nécessité comme suit:

1. Le besoin ésotérique interne, c'est-à-dire inhérent au micro-domaine, permettant l'échange et la discussion d'informations au sein même de l'hyperspécialisation, ce der-

Meta, XLVI, 1, 2001 
nier terme renvoyant tant aux chercheurs et aux praticiens qu'aux laboratoires pharmaceutiques travaillant sur un même protocole. La typologie discursive sera préférentiellement persuasive, aux fins conjuguées d'asseoir sa position et sa stratégie dans l'univers visé et d'obtenir des crédits pour financer telle ou telle recherche particulière.

2. Le besoin ésotérique externe, qui sort du micro-domaine pour s'adresser aux médecins généralistes ou d'autres spécialités. Il s'agit de les convaincre d'adopter une médication ou de prescrire un traitement spécifique. La typologie restera persuasive et s'accompagnera d'un effort d'information relayé par l'affirmation de la spécialité dans l'univers médical global.

3. Le besoin exotérique, visant essentiellement à informer de manière simple, claire et concrète un public extérieur à l'activité médicale, mais nécessairement en contact avec cette dernière par des consultations, des traitements ou d'une volonté d'information légitime. La typologie discursive sera de nature informative, axée sur la vulgarisation et, par conséquent, sur une simplification lexicale et cognitive de l'information fournie. Dans les notices pharmaceutiques, on remarquera un double discours, d'abord monosémique en ce qui concerne la composition du médicament, et ensuite plus général pour donner au patient des notions de confort et de clarté d'utilisation. On a donc en médecine, selon le destinataire, un lexique variable, plus ou moins marqué terminologiquement, et il faut bien reconnaître que rhume ne sera jamais tout à fait l'équivalent de rhinorrhée et que le public comprendra plus facilement déficit de plaquettes que thrombocytopénie.

En d'autres termes, on peut donc raisonnablement se rallier au commentaire de Régent (1992: 66) : «La communication scientifique écrite apparaît donc comme un réseau multidimensionnel de groupes restreints ou élargis qui communiquent entre eux, et aussi maintenant de plus en plus avec l'extérieur, par le biais de la vulgarisation. Les discours produits sont différents selon qu'ils circulent à l'intérieur d'un groupe restreint où tous les gens se connaissent et partagent la même information de base, ou dans des groupes plus larges dans la même spécialité, ou des groupes encore plus larges touchant plusieurs spécialités. Lorsque l'information de base n'est pas entièrement partagée, le discours doit être beaucoup plus explicite.»

\section{La notion de texte médical}

Toujours est-il qu'il convient de s'entendre sur la notion même de «texte médical», qui n'équivaut en aucune manière à celle de "texte écrit par un médecin ", même spécialisé. Pour illustrer cette problématique, dans quelle catégorie rangera-t-on le Cahier rouge de Luc Montagnier (1994: 49-51) qui narre l'isolement du virus du SIDA en 1983 ?

Pourquoi du sang? Pourquoi des ganglions? On sait à l'époque que le SIDA est une maladie qui détruit les lymphocytes T4 et qui est précédée de gonflements ganglionnaires qui peuvent persister des mois, voire des années. Il est donc logique pour moi de penser que l'agent du SIDA se trouve non seulement dans le sang, mais aussi dans les ganglions lymphatiques. Ces derniers sont en effet, avec la rate, le réservoir par excellence des lymphocytes.

[...]

Nous faisons alors des tentatives avec des lymphocytes d'autres donneurs, provenant en particulier de nouveau-nés. Mais le virus se multiplie sans jamais transformer les cellules. Je note avec soin tous nos résultats dans un cahier rouge, le même qui m’a servi depuis 1977 à la recherche de rétrovirus dans des cancers humains. À la page BRU, j'inscris «enfin» enfin quelque chose de solide. 
Ce texte est-il médical ? Certainement à en juger par la terminologie utilisée et le domaine auquel cette dernière renvoie. En outre, il est écrit par un médecin, un des plus célèbres immunologues de notre époque.

Pourtant, ce texte raconte une histoire, dont la trame, et déjà le titre, font penser à un roman policier. Du rififi dans les hôpitaux de Paris? Non. Simplement l'histoire d'un chercheur, dont les sentiments accompagnent inéluctablement les tâtonnements dans la quête d'un virus, traqué comme un malfaiteur. On se rend aisément compte que la dichotomie médecin/traducteur, qui fait régulièrement l'objet d'un traitement particulier dans tout ouvrage ou article traitant de traduction médicale, est peu pertinente, pour ne pas dire parfaitement aléatoire.

L'Histoire des Lettres nous montre par ailleurs le mirage de ce type de discours. François Rabelais n'était-il pas diplômé de la Faculté de médecine de Montpellier et médecin à l'Hôtel-Dieu de Lyon avant d'écrire son Gargantua et son Pantagruel, jalonnés d'érudition philologique et dont l'épisode de Panurge pose à merveille la problématique des langues? Le grand Émile Littré, connu essentiellement pour son Dictionnaire, féru en grec, en sanskrit et en arabe, ne fit-il pas des études de médecine avant de traduire les Euvres d'Hippocrate entre 1839 et 1861 ? Ce furent d'ailleurs ses traductions qui lui ouvrirent les portes de l'Académie française, plutôt que son œuvre de lexicographe.

\section{Langue commune et langue de spécialité}

La distinction récurrente entre langue générale et langue de spécialité, si chère à plusieurs auteurs, me semble utopique à en juger par la diversité discursive qui caractérise nombre de textes spécialisés, dont ceux relatifs à la médecine. La biunivocité, rêve de tout terminologue, reste un vœu pieux qui ne sera jamais exaucé. À ce propos, on lira avec intérêt les observations de Jean-Charles Sournia (1994: 693):

Ce vocabulaire oral, populaire, traduisait l'angoisse éternelle et universelle de l'homme devant son corps qu'il sait d'autant plus fragile qu'il en ignore le fonctionnement, constamment menacé par la maladie et donc la mort. De quelque science que se pare la médecine, elle ne supprime pas cette anxiété existentielle. Aussi cette terminologie reste le fondement de notre expression médicale; elle pèche par son imprécision, ses ambiguïtés (le mot gorge peut désigner plusieurs territoires anatomiques), ses particularismes même parmi les langues romanes (sein n'existe qu'en français), mais la médecine française moderne ne peut pas s'en affranchir. Elle découle d'une culture très ancienne, rudimentaire, naturaliste, qui ne doit rien ni au christianisme récent et encore fruste, ni à l'érudition latine des médecins universitaires.

Wimmer (1982: 17) distingue comme suit la langue scientifique et de spécialité, de la langue commune:

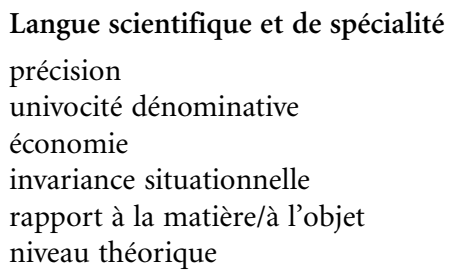

\author{
Langue commune \\ indétermination \\ ambiguïté \\ redondance \\ multiplicité situationnelle \\ multiplicité thématique/évaluation \\ quotidienneté
}


Kocourek (1982: 41) situe pour sa part le débat dans l'opposition objectivité/ subjectivité :

Elle [la langue de spécialité] vise l'idéal de l'intellectualisation, c'est-à-dire la précision sémantique, la systématisation conceptuelle, la neutralité émotive, l'économie formelle et sémantique; elle a donc tendance [...] à neutraliser ou à contenir l'émotivité, la subjectivité.

Comme nous le verrons plus avant, cette opposition ne résiste ni à l'épreuve des faits ni à celle des textes. La subjectivité est omniprésente dans la littérature médicale, et les tournures impersonnelles que l'on invoque à l'appui de la thèse objectiviste ne sont que le voile d'une subjectivité latente, inconsciente parfois, mais toujours insidieuse car occultée par le linguistique. Il incombe au traducteur de ne pas saisir ce qu'il y a sur la page mais ce qu'il y a dans le texte. Il convient dès lors de s'arrêter un moment sur la modalisation.

\section{La modalisation}

La médecine, comme les autres sciences, progresse par la remise en cause des théories précédentes ainsi que des auteurs du passé. La mise en doute est difficile, eu égard au prestige de ces auteurs et au caractère hypothétique des théories nouvelles. La prudence discursive est donc de rigueur et devra ménager l'objectivité, garante du caractère scientifique de l'écrit, et la subjectivité, destinée à mettre en avant le nouvel auteur par rapport aux confrères avec lesquels il débat. Myers (1989) accorde à la modalisation un statut de courtoisie, visant à ne pas discréditer les auteurs remis en question et à ne pas rompre avec la communauté médicale et ses modes de fonctionnement. J'ajouterais volontiers que la critique se pare, paradoxalement, des atours d'une reconnaissance envers les prédécesseurs, dont les travaux constituent un jalon essentiel des progrès modernes. La critique est aussi une manière directe de rendre hommage à autrui. La modalisation exprime enfin l'angoisse du praticien devant les aléas de la recherche et la caducité des affirmations péremptoires. L'analyse diachronique des découvertes précédentes préfigure déjà l'instabilité des certitudes nouvelles.

Le bref historique des psychoses périodiques maniaco-dépressives, retracé dans le classique Manuel de Psychiatrie d'Ey, Bernard et Brisset (1963: 232-233), en est un très bel exemple:

Si depuis l'Antiquité on avait noté des relations entre les accès de manie et de mélancolie, ces deux «crises» restèrent jusqu'au début du XIX ${ }^{\mathrm{e}}$ siècle des «maladies» séparées qui pouvaient seulement occasionnellement alterner ou se compliquer. Cette manière de voir était encore partagée par Pinel et par Esquirol. «Il n’est pas rare, écrivait ce dernier en 1816 , de voir la manie alterner d'une manière régulière avec la phtisie, l'hypocondrie et la lypémanie.»

[...]

Mais ce fut Kraepelin qui, en 1899, par la description de l'analyse minutieuse des états de transitions et les intrications des crises maniaques et mélancoliques aboutit à la notion d'états mixtes démontrant ainsi l'identité profonde de ces deux formes de troubles.

On sent en filigrane du texte une critique contenue, compréhensive, des prémices de la psychiatrie, avec dans le même temps une reconnaissance envers les pionniers de cette discipline inédite. L'extrait est aussi révélateur de deux indices qui témoignent 
de la mouvance de la discipline et de son caractère évolutif, non figé dans la monosémie d'un lexique immuable:

1. La variété dénominative. La phtisie, «maladie du $\mathrm{XIX}^{\mathrm{e}}$ siècle », est devenue au $\mathrm{xx}^{\mathrm{e}}$ siècle la tuberculose. Ce n'est ni l'affection ni le concept qui ont évolué, c'est tout simplement la dénomination, sujette aux avatars sociohistoriques.

2. Le caractère erratique de la composition scientifique des termes médicaux. La psychiatrie admet et conserve sans rechigner hypochondrie aux côtés d'hypocondrie. De même, cholédoque coexiste avec mélancolie, alors que la racine grecque kholè (la bile) est commune aux deux termes. Peut-être parce que mélancolie («bile noire») participe autant du vocabulaire courant que du vocabulaire psychiatrique. C'est sans doute dans ce sens qu'on peut expliquer la graphie du mot colère, qui, à l'instar de choléra, renvoie au même étymon.

\section{La terminologie médicale}

L'opinion de Nakos (1994-1995: 269) conforte le mirage de l'objectivité terminologique:

On pourrait prétendre que l'image [appliquée à la langue spécialisée] manque de précision, qu'elle est ambiguë et que la création de termes définitoires monosémiques pallie ces inconvénients. Encore faudrait-il que ces nouveaux termes non connotés puissent rapporter adéquatement la réalité, ce qui ne peut être le cas de façon absolue car un terme définitoire n'arrive jamais à décrire une réalité ou une situation dans son intégralité. En outre, le principe de monosémie est utopique: la richesse d'une langue provient justement du fait que les expressions évoluent et prennent de nouvelles acceptions. La langue est dynamique et ne peut s'accommoder du caractère artificiel et statique de la monosémie qui, poussée à l'extrême, encombre inutilement la langue et finit par l'appauvrir en l'enfermant dans un carcan.

On remarquera par conséquent que la terminologie médicale, marquée du sceau de l'érudition, ne garantit en aucune manière la vérité ni la précision du discours. En effet, quelle est donc cette science qui, dans ses assertions, fait d'un anémique un exsangue et d'une tumeur cervicale un cancer du cou?

Cette distinction, toute relative, entre vocabulaire courant et savant, est un reliquat historique, non imputable à l'incompétence scientifique des couches populaires, mais imposée pour légitimer la profession médicale aux $\mathrm{XVI}^{\mathrm{e}}$ et $\mathrm{XVII}^{\mathrm{e}}$ siècles, par rapport aux autres membres de la société, mis au ban d'un savoir qui ne saurait être partagé.

La naissance de la terminologie médicale en langue française au $\mathrm{XvI}^{\mathrm{e}}$ siècle, qui servira la quête de la biunivocité si prisée dans le domaine scientifique, s'inscrit dans un sociolecte indolore où la logorrhée pseudo-savante s'échine à dédouaner l'incapacité du praticien sur le terrain. La terminologie savante sort du cadre muet des dictionnaires pour être proférée à l'adresse du patient et détourner le pôle d'attention du malade vers le médecin.

Ce sont précisément ces médecins, dont la vanité le dispute à l'incompétence, que nous retrouvons dans les savoureuses caricatures de Molière. Drapés dans leur longue robe noire, les Purgons et Diafoirus occultent leur ignorance sous un continuel verbiage, mi-français, mi-latin, entrecoupé de nombreuses citations d'Hippocrate et de Galien. Tâtant le pouls d'une main hautaine et examinant le flacon 
d'urine de l'autre, ils terminent leur consultation par une formule cryptique et un bon clystère. Relisons pour le plaisir un court extrait du Malade imaginaire (acte III, scène III) :

ARGAN - Les médecins ne savent donc rien, à votre compte?

BÉRALDE - Si fait, mon frère. Ils savent la plupart de fort belles humanités, savent parler en beau latin, savent nommer en grec toutes les maladies, les définir et les diviser; mais, pour ce qui est de les guérir, c'est ce qu'ils ne savent point du tout.

ARGAN - Mais toujours faut-il demeurer d'accord que sur cette matière les médecins en savent plus que les autres.

BÉRALDE - Ils savent, mon frère, ce que je vous ai dit, qui ne guérit pas de grand-chose, et toute l'excellence de leur art consiste en un pompeux galimatias, en un spécieux babil, qui vous donne des mots pour des raisons et des promesses pour des effets.

Pour en revenir au Cahier rouge de Montagnier (1994), si nous analysons les modalités discursives mises en ouvre dans le passage cité, nous pouvons effectuer quelques observations intéressantes, regroupées sous les deux rubriques qui suivent.

\section{Conjonction lexicale entre termes de spécialité et vocabulaire courant:}

- Les termes de spécialité sont bien entendus fréquents, qu'il s'agisse de lymphocytes, immunodépression, bec Bunsen, hottes à flux laminaire, staphylocoques, interleukine 2, transcriptase inverse, $H T L V$, etc. Cela prouve sans conteste que l'extrait renvoie à un domaine de spécialité bien circonscrit, un micro-domaine, en l'occurrence celui de l'immunologie. D'autre part, une étude un peu plus précise des occurrents montre qu'il s'agit d'immunologie appliquée au SIDA, et plus particulièrement de démarche biologique et non clinique. Enfin, le texte renvoie à une communauté d'initiés, médecins ou non, mais qui sont en tout cas familiarisés avec le domaine dont il est question. Le livre dont est extrait le Cahier rouge et qui s'intitule Des virus et des hommes est publié aux Éditions Odile Jacob, ce qui situe d'emblée les destinataires ciblés.

- Le vocabulaire courant est également à l'honneur, avec des mots ou des syntagmes comme volume, origine, cause, à la nuit tombée, cauchemar, fructueuse, transformer, cahier, quelque chose... Rien dans ces mots ou syntagmes, et je pourrais allonger la liste, ne prédispose à penser que nous sommes en présence d'un texte médical, et non généraliste de surcroît. On admettra dès lors qu'un texte spécialisé se doit, pour des raisons d'articulation syntaxique et sémantique, de recourir à un stock lexical et stylistique dont la fonction est tant descriptive qu'explicative et conjonctive.

\section{Conjonction entre objectivité scientifique et subjectivité explicite:}

- La caution du caractère scientifique du texte et donc de la démarche de l'auteur se doit d'apparaître tout au long de l'écrit. La rigueur de l'enchaînement logique, indubitablement marquée au coin de l'univocité terminologique, doit cautionner l'exactitude de l'écrit. Le passage suivant est fort éclairant:

Une partie est congelée à $-801 / 2 \mathrm{C}$ pour en extraire plus tard l'ADN. L'autre est mise en culture en présence d'une fraction protéique provenant du staphylocoque, la protéine A, capable d'activer les lymphocytes. En induisant une multiplication cellulaire active, il 
s'agit de faire «sortir» le rétrovirus, supposé persister à l'état latent dans certains lymphocytes. Mais je ne sais pas lesquels. S'agit-il des lymphocytes B ? Des lymphocytes T? Pour l'instant, je place les deux flacons étiquetés BRU 3.01.83 et contenant des cultures de lymphocytes de sang et de ganglion en chambre chaude maintenue à $371 / 2 \mathrm{C}$, condition de croissance idéale. Le 6 janvier, pour nourrir mes cultures, j'ajoute des facteurs de croissance des lymphocytes $\mathrm{T}$, de l'interleukine 2 , ainsi que du sérum anti-interféron.

Quelle rigueur et quelle précision dans le déroulement des opérations! Le questionnement sur l'activité humorale ou cellulaire, l'étiquetage des flacons, les températures de chauffage, l'adjonction d'IL2, autant de garanties sur la valeur des observations. Le lecteur suit in vitro la séquence opératoire et ne fait l'impasse sur aucun enchaînement. Tout est vérifiable.

- La subjectivité, non seulement inconsciente mais aussi parfois consciente, affleure aussi dans le texte, notamment dans l'interprétation du résultat. Relisons la fin de l'extrait:

Les lymphocytes d'un donneur, un Espagnol de passage qui se présente ce jour-là, s'avèrent excellents. Nous les mélangeons avec ce qui reste des lymphocytes de BRU. [...] Je note avec soin tous nos résultats dans un cahier rouge, le même qui m’a servi depuis 1977 à la recherche de rétrovirus dans des cancers humains. À la page BRU, j'inscris «enfin» enfin quelque chose de solide.

Quel sens donner par exemple à la dernière phrase? Qu'y a-t-il de plus subjectif que de lire "enfin» ou quelque chose de solide? Comment comprendre le sens du mot excellents?

La forme impersonnelle, tant soulignée chez les commentateurs de textes scientifiques, ne résiste pas non plus à la simple lecture du texte. Que penser de un Espagnol de passage ou de je note? Quel est ce Nous de Nous les mélangeons? S'agit-il de l'équipe de Montagnier, de Montagnier et de Eyquem, son collègue de l'Institut Pasteur, ou de l'ensemble de la communauté scientifique préoccupée par le sujet à cette époque?

Et pourtant, la subjectivité fait à mon sentiment partie intégrante de la démarche scientifique. Le rôle fondamental de l'intuition dans la recherche est indéniable, tant il est vrai que l'on trouve avant tout ce que l'on cherche. L'intuition n'est-elle pas la clef de voûte des travaux de Jenner et de Pasteur, pour ne citer qu'eux, sans laquelle la vaccination aurait tardé à s'imposer? Toutes les découvertes participent de la vérification empirique d'un postulat de départ, dont le caractère aléatoire n'est que relatif, puisqu'il s'arc-boute sur la domination d'un savoir vérifié.

\section{Importance de l'initiation et de l'actualisation}

On peut s'être spécialisé dans un domaine ou même un micro-domaine sans pour autant connaître les arcanes de la profession au quotidien. C'est ainsi que le travail de biologiste en laboratoire ne rejoint pas nécessairement, loin s'en faut, celui du clinicien en structure hospitalière. Il y a par voie de conséquence une initiation qui n'est plus à proprement parler d'ordre scientifique, mais qui a trait à la pratique quotidienne de tel ou tel protocole particulier mené à l'intérieur d'une structure bien définie.

Dans l'extrait cité, même un immunologue averti peut rester à quia devant certaines données du texte. Ainsi, comment comprendre BRU 3.01.83, même s'il est 
clair de par le contexte que les chiffres renvoient à la date de l'essai? Il s'agit véritablement d'un code, puisque dans d'autres recensions on pourra trouver la mention RUB. Savoir qu'il s'agit de l'apocope du nom Brugière, celui du patient dont on a prélevé l'échantillon sanguin, aidera non seulement à la compréhension du passage, mais aussi à un gain de temps énorme dans le décryptage du sens. La connaissance du domaine dépasse largement le simple cadre théorique pour rejoindre, dans de nombreux cas, la pratique professionnelle quotidienne.

\section{La traduction des textes médicaux}

Selon Ladmiral (1979: 11), la traduction est «un cas particulier de convergence linguistique: au sens le plus large, elle représente toute forme de médiation interlinguistique permettant de transmettre de l'information entre locuteurs de langues différentes. La traduction fait passer un message d'une langue de départ (langue-source) à une langue d'arrivée (langue-cible).» Cette définition, comme tant d'autres, situe la traduction dans le champ de la linguistique et voit dans la conjonction de deux langues la condition indispensable à tout acte de traduction.

D’après Hurtado Albir (1990: 57-63), la traduction est un «acte de communication" qui vise à faire comprendre un texte écrit (ou un discours oral) à un destinataire qui ne comprend pas la langue, voire la culture, de l'original. Il s'agit par conséquent d'une activité intertextuelle et non interlinguistique, dont participent tant les éléments linguistiques (connaissance des langues) que les éléments extralinguistiques (connaissance de la situation, du sujet, de la civilisation, etc.), ce qui signifie que, ce que l'on traduit, ce ne sont pas les mots ni les structures syntaxiques (corrélation paradigmatique des mots) mais le sens. En d'autres termes, traduire, c'est reconstituer cognitivement le vouloir-dire de l'auteur.

Si l'on ne peut que rejoindre l'avis de Hurtado Albir, force est de constater que la présence de deux langues conditionne l'acte de traduction. Et pourtant, l'autotraduction existe, et particulièrement dans un domaine comme la médecine. Sur quel fondement théorique s'appuyer alors pour l'étudier?

\section{L'autotraduction dans le domaine des publications médicales}

La notoriété internationale souhaitée par les chercheurs qui publient leurs découvertes ou les résultats cliniques des protocoles engagés justifie le recours à l'autotraduction, car la rédaction directe dans une langue étrangère ne saurait faire l'économie des modes de pensée et des modèles linguistiques de la culture de départ.

En outre, la volonté et le besoin pressant de communiquer dans les meilleurs délais et à l'échelle planétaire les bénéfices des recherches menées, qu'elles le soient en laboratoire, en milieu hospitalier ou dans le cadre d'une firme pharmaceutique, poussent les scientifiques à publier directement dans les revues de prestige, dont la langue véhiculaire est l'anglais. C'est ainsi qu'à une certaine époque, l'Institut Pasteur arrêta la publication de ses Annales en langue française. On en conviendra, les exemples d'autotraduction, au sens où je viens de la définir, sont rarissimes dans le domaine littéraire et ne font qu'exception, comme Nabokov et son Lolita, pour citer un cas. 


\section{Traduction et connotation}

J'ai déjà montré ailleurs (Balliu 1994) la dilution de la notion de connotation chez des linguistes aussi prestigieux que Mounin ou Martinet. Celui-ci (1969: 342) ramène l'opposition dénotation-connotation à la distinction entre la fonction référentielle et la fonction émotionnelle des signes. Cependant, si les textes de la langue spécialisée font appel à la connotation, c'est précisément parce qu'ils privilégient le sens. C'est ainsi que l'étudiant devra se familiariser avec les tours propres à la littérature médicale, justiciables de la sociocritique et dont les implications sont extralinguistiques. Le domaine de la thérapeutique sera friand d'expressions comme "arsenal thérapeutique», «batterie de tests», «traitement d'attaque» ou «population cible». De même, les patients constitueront des «cohortes» et seront "enrôlés» dans des protocoles qui détermineront par exemple les «colonies» de lymphocytes. Les différents paramètres recueillis permettront de définir des «stratégies thérapeutiques». Ces tours relèvent du vocabulaire militaire et visent à asseoir le potentiel thérapeutique du praticien dans son rapport à la maladie.

En d'autres termes, la littérature scientifique n'est en aucun cas le degré zéro de la connotation; la seule différence avec les œuvres littéraires réside dans le fait que la connotation y est plus pernicieuse, comme si elle désavouait le caractère objectif de l'écrit.

Il est par ailleurs curieux et intéressant de relever que nombre d'ouvrages sur la traduction médicale, qui se veulent à la fois méthodiques et pratiques, n’accordent que peu ou pas de place à l'étude de la connotation, pourtant tellement importante dans le domaine médical. Un ouvrage assez complet comme celui de Rouleau (1994), qui contient nombre d'informations utiles, n'analyse pas ce problème crucial et fait l'impasse sur le sociolecte de la profession (Balliu 1995).

Or, la médecine est une pratique plurimillénaire, exercée par l'homme sur l'homme, et les considérations sociolinguistiques qu'elle véhicule sont omniprésentes dans le langage utilisé. Pour donner un exemple, les liens conflictuels qui unissent le médecin à la maladie ont généré un foisonnement de termes et d'expressions intrinsèquement étrangers au domaine, mais qui se sont imposés pour traduire dans la langue une efficacité parfois plus douteuse, voire inexistante, sur le terrain.

Voici une illustration de ce phénomène à travers six extraits tirés d'un article de Marchal (1993) publié dans La Recherche et consacré au réveil de la tuberculose:

1. Là, ils sont rapidement inactivés par des cellules immunitaires, les macrophages, qui ont la capacité d'ingérer les bactéries puis de les détruire grâce à une batterie d'enzymes.

2. Ce milieu est très favorable au développement, maintenant extracellulaire, des bacilles dont le nombre atteint rapidement dix millions à plus de un milliard en quelques semaines. Ils colonisent d'autres territoires pulmonaires et éventuellement le tube digestif.

3. Une analyse épidémiologique fine, effectuée en 1991 par K. Brudney et J. Dobkin [...] a montré que la population cible des deux infections se recoupait.

4. Aux États-Unis, l'augmentation des cas de tuberculose et des souches résistantes a été imputée dans un premier temps au "couple maudit» formé par le sida et la tuberculose, selon la formule utilisée par le médecin français J. Chrétien.

5. De rechutes en traitements anarchiques, les souches multi-résistantes sont sélectionnées avec une fréquence d'autant plus grande que la population considérée est pauvre.

6. Les molécules efficaces sont donc soit sécrétées par la bactérie vivante [...] soit démasquées lorsque la bactérie se divise. 


\section{Dépersonnalisation du patient par le médecin}

Dès le $\mathrm{XVI}^{\mathrm{e}}$ siècle, la formation de la terminologie savante ne pouvait faire l'impasse sur l'appropriation du concept et du référent par la profession médicale. Ce point me semble essentiel. Cela est dû au fait qu'au xvi ${ }^{\mathrm{e}}$ siècle le savoir médical sort des livres pour pénétrer, grâce aux progrès de l'anatomie et à l'autorisation de la dissection, dans un corps fait de chair et de sang auquel, singulièrement, le propriétaire n'a pas accès.

Seul le médecin appréhende les arcanes du corps et c'est à la Renaissance que l'on doit le développement des éponymes médicaux qui mettent les médecins sous les feux de la rampe, en reléguant dans l'ombre le malade dépositaire de la maladie, à qui on dénie information et vérité. C'est ainsi que Fallope (1523-1562), ancien chanoine de la cathédrale de Modène, donna son nom aux trompes de l'utérus et au ligament de l'arcade crurale. Bartholomé Eustache (1520-1574), médecin du pape et professeur à l'Université, restera à jamais l'homme de la trompe et de la valvule de la veine cave inférieure qui portent son nom.

Ce même phénomène rebondit au XIX ${ }^{e}$ siècle avec le triomphe de la médecine clinique qui repose sur l'observation séméiologique du patient. L'exploration manuelle du corps et les techniques d'auscultation renforcent le pouvoir du médecin sur l'anatomie et la physiologie du malade. C'est ainsi que les éponymes enregistrent un nouvel essor en médecine et que les pathologies qu'ils désignent portent toujours le nom du médecin qui les a découvertes ou guéries, jamais celui du malade qui en a été atteint. Songeons à la maladie d'Alzheimer, aux bacilles de Hansen et de Koch, au syndrome de Korsakov... Une étude plus détaillée de ce phénomène montrerait que le sociolecte médical n'est pas l'apanage de la seule terminologie.

\section{Le traducteur médical}

On se rend aisément compte, à la lumière de l'analyse qui précède, que le texte, sous son apparence figée, recèle nombre de mystères imperceptibles à la simple observation superficielle. Le traducteur, pour déceler les clefs qui ouvrent les portes de la compréhension, devra démasquer le texte, le disséquer et faire œuvre de médecin légiste pour en retracer l'histoire dans toute sa vérité.

La maîtrise des sources d'informations, fondée notamment sur la connaissance de la terminologie et l'accès aux bases de données documentaires, ne suffira en aucune manière à autoriser une traduction fiable, dans la mesure où un texte bien documenté n'est pas forcément un texte bien traduit.

C'est la connaissance approfondie du domaine, le contact chronique avec les praticiens et la littérature médicale, surtout en langue d'arrivée, qui permettront au traducteur de bien comprendre et de bien rendre le texte original et d'y amender, le cas échéant, les erreurs qu'il y aura repérées.

Le savoir médical, plus que tout autre, est fait d'échanges permanents, tant pratiques que théoriques, entre les différents acteurs que sont les thérapeutes et les patients. Ce savoir évolue très rapidement et est tributaire à l'extrême des variations géographiques, culturelles et temporelles. Les dénominations et modes d'expression véhiculés par les différents acteurs oscillent au gré des circonstances. 
Traduire va donc bien au-delà du simple exercice interlinguistique ou terminologique pour plonger au cœur même de la vie et de sa manifestation discursive. Les référents, les champs notionnels ou encore l'étude des collocations ne permettent de visualiser que la pointe de l'iceberg textuel, sans en dévoiler la structure la plus profonde. Comme l'écrivait Claude Bernard dans son Introduction à l'étude de la médecine expérimentale (1865): « $S$ 'il fallait tenir compte des services rendus à la science, la grenouille occuperait la première place.»

\section{RÉFÉRENCES}

Balliu, C. (1994): «L'enseignement de la traduction médicale: pour une nouvelle pragmatique», Meta, 39-1, p. 15-25.

- (1995): compte rendu de M. Rouleau (1994): «La traduction médicale, une approche méthodique», Meta, 40-4, p. 656-658.

Ey, H., P. Bernard et C. Brisset (1963): Manuel de psychiatrie, Paris, Masson.

Hurtado Albir, A. (1990): La notion de fidélité en traduction, Paris, Didier Érudition.

Kocourek, R. (1982): La langue française de la technique et de la science, Wiesbaden, Brandstetter.

Ladmiral, J.-R. (1979): Traduire: théorèmes pour la traduction, Paris, Payot, coll. «Petite Bibliothèque Payot».

Lock, S. (1989): “'Journalology': Are the Quotes Needed?", CBE Views, 12-4.

Marchal, G. (1993): «Le réveil de la tuberculose», La Recherche, 253, p. 380-388.

Martinet, A. (1969): La linguistique, Paris, Denoël.

Montagnier, L. (1994): Des virus et des hommes, Paris, Odile Jacob.

Myers, G. (1989): "The Pragmatics of Politeness in Scientific Articles", Applied Linguistics, 10-1.

Nakos, D. (1994-1995): «Les images en médecine: une perception vive et précise des réalités», Actes de langue française et de linguistique, 7/8, p. 269-282.

RÉGENT, O. (1992): «Pratiques de communication en médecine: contextes anglais et français», Langages, 105, p. 66-75.

Rouleau, M. (1994): La traduction médicale, une approche méthodique, Montréal, Linguatech.

SourniA, J.-C. (1994): «Les phases évolutives du vocabulaire médical français», Meta, 39-4, p. 692-700.

Wimmer, R. (1982): «Wissenschaftliche Kommunikation und Gemeinsprache», WissenschaftSprache-Gesellschaft, Rehburg-Loccum, p. 15-32. 